\title{
El profesionalismo en la formación del médico en la Universidad Nacional Mayor De San Marcos. Un estudio de sílabos
}

\author{
Professionalism in medical education at Universidad Nacional Mayor de San Marcos. A
} study of syllabi

\author{
Cecilia Sogi ${ }^{1, a}$, Maximiliano Cárdenas ${ }^{1, a}$, Salomón Zavala ${ }^{1, a}$, Alfonso Mendoza $^{1, a}$, \\ María del Rosario Jiménez $z^{1, b}$ \\ Facultad de Medicina, Universidad Nacional Mayor de San Marcos, Lima, Perú. \\ ${ }^{a}$ Instituto de Ética en Salud; ${ }^{b}$ Departamento de Medicina Comunitaria.
}

\begin{abstract}
Resumen
Objetivo. Identificar la enseñanza del profesionalismo (excelencia, humanismo, responsabilidad y altruismo) en el plan de estudios médicos de la Universidad Nacional Mayor de San Marcos. Diseño. Estudio cualitativo, específicamente, el cifrado cualitativo. Institución. Facultad de medicina, Universidad Nacional Mayor de San Marcos, Lima, Perú. Material: Silabos de plan de estudios médicos. Métodos. Se examinó 22 silabos de un total de 38 correspondientes al plan de estudios médicos del primero al sexto año. Se conformó un panel de expertos y se optó por el método de análisis de contenido. Se estableció la confiabilidad inter-evaluador y se identificó los principios del profesionalismo en las competencias, contenidos, estrategias metodológicas y evaluación de los sílabos. Principales medidas de resultados. Enseñanza del profesionalismo. Resultados. El $58 \%$ de los 38 cursos aludió a algún principio del profesionalismo y, de estos, las competencias relacionadas con excelencia fueron las más frecuentes, seguidas de humanismo y responsabilidad. Los contenidos no se refirieron al profesionalismo, excepto en algunos. Las estrategias metodológicas de algunos cursos mencionaban explícitamente modelos de rol del docente y el aprendizaje de la conducta profesional en la práctica clínica. La evaluación era a través de exámenes cognoscitivos y calificación del docente que evaluaba la práctica y otras actividades del alumno. Conclusiones. Los principios de excelencia, humanismo y responsabilidad estuvieron presentes en mayor medida en las secciones de competencias y estrategias metodológicas, y en menor medida en contenido y evaluación del curso.

Palabras clave. Educación; Evaluación de la Enseñanza; Escuelas Médicas; Currículo médico; Universidad Nacional Mayor de San Marcos.
\end{abstract}

\begin{abstract}
Objective. To identify teaching of professionalism (excellence, humanism, accountability and altruism) in the medical curricula of the Universidad Nacional Mayor de San Marcos. Design. Qualitative, specifically encoded qualitative study. Setting. Faculty of Medicine, Universidad Nacional Mayor de San Marcos, Lima, Peru. Material. Medical education syllabi. Methods. Thirty-eight syllabi of courses corresponding to the six years of medical education were examined. A panel of experts was settled and the content analysis method was used. The inter-rater reliability was established and principles of professionalism were identified in competencies, contents, teaching methods and syllabi assessment. Main outcome measures. Teaching of professionalism. Results. Fifty-eight per cent of courses mentioned some elements of professionalism; the most frequent competencies were related to excellence followed by humanism and accountability. Contents did not refer to professionalism except in a few courses. The teaching methods of some courses explicitly mentioned teachers' role model and learning of professional conduct in clinical practice. Evaluation was through cognitive assessment and teachers' score assessing students' practice and other activities. Conclusions. Excellence, humanism and accountability were present more frequently in competence and teaching methodology and less frequently in content and course assessment.
\end{abstract}

Keywords. Teaching; Educational Measurement; Schools, Medical; Curriculum, Medical; Universidad Nacional Mayor de San Marcos.

An Fac med. 2015;76(4):339-48 / http://dx.doi.org/10.15381/anales.v76i4.11403

\section{INTRODUCCIÓN}

El escenario del ejercicio médico viene cambiando drásticamente, de un lado, por las características de la medicina moderna altamente tecnificada $y$, de otro, por las formas de organización de los servicios de salud. En este escenario, Reed y Evans (citados por Hensel y Dickey) identificaron, hace más de dos décadas, un problema emergente que denominaron desprofesionalización del profesionalismo de la medicina, para referirse a tendencias en la atención de salud que limitan la autonomía profesional de los médicos y sitúa a la medicina en riesgo de degenerar de una profesión a un negocio ${ }^{(1)}$. En el país, Perales y cols., en el artículo El mercado profesional como determinante de inconducta médica advierte sobre el mismo fenómeno ${ }^{(2)}$.

Desde la década de los noventa, diversas instituciones médicas representativas han tomado iniciativas para promover y reforzar un ejercicio médi- co que enfrente los retos del cambiante escenario sin perder y, a su vez, afianzar los valores profesionales. El American Board of Internal Medicine (ABIM) impulsó el proyecto profesionalismo en el año $1995^{(3)}$. Posteriormente, en 2002, la Federación Europea de Medicina Interna, conjuntamente con el American College of Physicians, la American Society of Internal Medicine y el ABIM, adoptaron un estatuto para el ejercicio de la medicina, basado en los principios de la bioética ${ }^{(4)}$. 
El relanzamiento del profesionalismo tiene un espacio importante en la formación de los nuevos médicos. Autores como Burack y cols. afirman que la esencia de la educación profesional del médico incluye transmitir no solo conocimiento sino también valores y actitudes de una generación a otra ${ }^{(5)}$. Otros, como Relman, sostienen que hay que preparar mejor a los estudiantes y evitar que las escuelas gradúen técnicos altamente calificados para la industria de los servicios médicos ${ }^{(6)}$. Hay urgencia en afrontar la tarea de rediseñar la educación médica de forma tal que dote a los futuros profesionales de las competencias que les permitan actuar en escenarios cambiantes. En nuestro medio, Risco señala que: "los cambios que las instituciones formadoras de profesionales de salud deben realizar son de tal magnitud que implica una segunda reforma de la educación en salud equivalente a la primera reforma, producida en base al informe Flexner en el 1910" (7).

En el ámbito de la educación médica norteamericana, una de sus figuras representativas, Jordan Cohen, en su artículo Leadership for medicine's promising future (1998) plantea lo siguiente: "la calidad de la educación médica se debe evaluar no solo examinando cómo se lleva a cabo sino también examinando aquello que produce, es decir, las características del profesional que forma. Para ello las escuelas deben establecer objetivos precisos y medibles de la educación, objetivos que se correspondan con: 1) las expectativas legítimas de la sociedad; y, 2) los preceptos tradicionales de la educación médica. Respecto a lo primero, en el siglo 21 los médicos deben: a) ser altruistas, compasivos y empáticos en la atención de pacientes y, al mismo tiempo, confiables y honestos en todas sus relaciones sociales; b) comprender la base científica de la medicina y ser capaces de aplicar esa comprensión al ejercicio de la medicina; c) ser altamente diestros en brindar una atención adecuada a pacientes individuales basada en la mejor evidencia de eficacia disponible; d) ser capaces y sentirse en el deber de colaborar con otros profesionales de la salud y hacer uso de aproximaciones sistemáticas para promover, mantener y mejorar la salud individual y colectiva. Además, los preceptos de la profesión médica que debemos inculcar a los estudiantes son: altruismo, respeto, compasión, honestidad e integridad" ${ }^{(8)}$.

En 1999, la Association of American Medical Colleges (AAMC) publicó el Medical School Objective Project (MSOP), una declaración de consenso que establece cuatro atributos clave que debe adquirir el estudiante de medicina: altruismo, conocimientos, habilidades y vocación de servicio ${ }^{(9)}$. En el mismo año, el Accreditation Council on Graduate Medical Education (ACGME) adoptó seis competencias generales para médicos residentes, uno de los cuales es el profesionalismo, considerado esencial para las restantes cinco: atención de pacientes, habilidades de relación y comunicación, conocimiento médico, mejora en base a la experiencia y práctica basada en sistemas ${ }^{(10)}$.

Un modelo de profesionalismo que no es meramente la ausencia de atributos negativos sino más bien un conjunto de cualidades o conductas positivas es una propuesta de Baldwin y Daugherty. Un verdadero profesional médico evitará hacer el mal (no maleficencia), pero también buscará hacer el bien (beneficencia). En este modelo, el verdadero profesional médico alcanzará los mejores resultados posibles y utilizará los mejores medios o métodos. Así, el profesionalismo implica una base de competencia clínica, comprensión ética y habilidades de comunicación sobre las que se erige la aspiración a la aplicación juiciosa de los principios humanistas, que satisface las necesidades de atención de la salud de pacientes y de la comunidad ${ }^{(11)}$.

\section{Evaluación del profesionalismo}

La necesidad de la formación profesional del estudiante y residente de medicina conduce inevitablemente hacia temas de su medición y evaluación.
Shrank y cols., tomando la afirmación de Cohen de que "la calidad de la educación médica debe ser evaluada por aquello que produce", plantean que los próximos pasos deben encaminarse más allá del proceso educativo e incidir en el desarrollo y las mejoras continuas en los métodos para evaluar resultados y hacer acopio de evidencias sobre las mejores prácticas orientadas a lograr las metas deseadas ${ }^{(12)}$.

Por su lado, el Accreditation Council for Graduate Medical Education (ACGME), desde 1999, cambió el foco de la acreditación, de estructuras y procesos hacia los resultados de la educación y cambió la pregunta ipuede educar? por ¿cuál es la evidencia de que ha educado bien? ${ }^{(13)}$

No obstante la preocupación y el interés para evaluar profesionalismo y humanismo, existen serias dificultades para llevarlas a cabo. En una revisión, Misch cita la afirmación de ABIM y otros de que "la observación directa de la conducta del médico probablemente es el método más confiable, válido y útil para evaluar profesionalismo y humanismo". Señala que de las diversas técnicas, instrumentos y evaluadores, las que más se usa son las escalas de evaluación completadas por médicos docentes. Sin embargo, existe escepticismo sobre los mismos expuesto por Richards y cols.: "Estos formatos de evaluación clínica están plagados de falta de confiabilidad y error de generosidad - esto es, la mayoría de los sujetos son calificados con los puntajes más altos del instrumento-. Existe ambigüedad en los ítems y a menudo se observa un efecto de halo -esto es, una tendencia del evaluador a calificar a un estudiante de modo similar en todas las categorías evaluadas-". El autor citado comparte la opinión de otros de que el método cuantitativo no es la única aproximación a la obtención e interpretación de datos. La evaluación cualitativa, aunque considerada 'blanda', poco confiable, de escaso valor práctico y subjetiva, no significa que no sea válida, precisa ni tampoco poco confiable ${ }^{(14)}$. 
Por su lado, Arnold, luego de una extensa revisión de la literatura sobre profesionalismo concluye cuestionando la eficacia de los esfuerzos orientados a educar estudiantes en lo que es conducta profesional sin antes contar con instrumentos de evaluación consistentes ${ }^{(15)}$.

\section{Estudios empíricos}

En la literatura norteamericana existen estudios sobre la enseñanza del profesionalismo en las escuelas de medicina. Swick y cols., llevaron a cabo una encuesta a 125 decanos y directores de escuelas de medicina, en 1998, y hallaron que su enseñanza varía ampliamente y, aunque la mayoría de las escuelas lo consideran importante, las estrategias utilizadas en su enseñanza no serían las adecuadas ${ }^{(16)}$.

De los diversos estudios sobre métodos de evaluación del profesionalismo, entre ellos, Woolliscroft y cols. hallaron que pacientes, médicos asistentes, supervisores de programa y enfermeras perciben de modo diferente los atributos humanistas de residentes en su interacción con pacientes. Lo importante de los hallazgos fue que se requiere de diez a veinte enfermeras para una evaluación confiable del humanismo y que para obtener el mismo nivel de confiabilidad se necesitan de cincuenta médicos asistentes y más de cien pacientes ${ }^{(17)}$.

Por su lado, Hemmer y cols. compararon tres métodos de evaluación del profesionalismo en estudiantes del tercer año y hallaron que las deficiencias severas que necesitaron de intervención correctiva fueron más probables de ser identificadas en ambientes de hospitalización que en consultorios externos. De los tres métodos, las sesiones de evaluación formal cara a cara, a diferencia de la lista de chequeo y los comentarios escritos, mejoró la detección de la conducta no profesional en campo clínico ${ }^{(18)}$.

La importancia del tema motivó a un grupo de investigadores del Instituto de Ética en Salud de la Facultad de Medicina de la UNMSM a emprender una línea de investigación sobre la enseñanza del profesionalismo y su evaluación en la formación médica de pregrado. El presente es un estudio exploratorio preliminar que indaga sobre la presencia del profesionalismo en los sílabos del plan de estudios médicos.

La transcripción expresa el propósito de la Facultad de Medicina de la UNMSM de educar a los futuros profesionales de la salud en los valores que definen el profesionalismo médico: "Somos la comunidad académica de la Universidad Nacional Mayor de San Marcos, dedicada a formar profesionales de la salud, capacitar especialistas y prepararlos para la docencia e investigación, buscamos la verdad e interactuamos activamente con inclusión de la diversidad y la pluriculturalidad. Nuestros profesionales del más alto nivel técnico y científicos, son integrales, éticos, humanistas, críticos, respetuosos de los derechos humanos y el medio ambiente".

Fue objetivo del estudio identificar si los valores inherentes al profesionalismo médico (excelencia, responsabilidad, humanismo y altruismo) están presentes en los sílabos de los cursos del plan de estudios médicos de la UNMSM.

\section{MÉTODOS}

Con relación a la definición de profesionalismo, en el año 2006, Stern publicó el libro Measuring medical professionalism en el cual, junto con Arnold, propusieron una definición que plantea que los principios de excelencia, humanismo, responsabilidad y altruismo son elementos necesarios y suficientes para definir profesionalismo, considerando además que estos deben asentar sobre una base de competencia clínica, habilidades de comunicación y comprensión ética y legal ${ }^{(19)}$. Las conductas observables que se presenta en el anexo ilustra la aplicación de los principios mencionados.

El ámbito del estudio fue la Facultad de Medicina de la UNMSM. El plan de estudios de la Escuela Académico Profesional de Medicina Humana (EAPMH) comprende un total de 42 cursos distribuidos en siete años de estudio. Se excluyó cuatro cursos del sétimo año que corresponden al internado, etapa de formación eminentemente práctica, razón por la que consideramos amerita tratarse en otro estudio. Se hizo acopio de los sílabos de cursos del primero al sexto año correspondientes al año académico 2013.

Se examinó los 38 cursos, separándose del estudio 16 porque su contenido solo mencionaba aspectos eminentemente técnicos. Así, el material consistió en una fuente de información secundaria consistente en 22 sílabos.

Para el estudio se utilizó el enfoque cualitativo, específicamente, el cifrado cualitativo, es decir, se organizó y analizó datos que originalmente no fueron creados para fines de la investigación. El término se aplica a todas las técnicas destinadas a una clasificación fidedigna de los datos sociales. Los pasos fueron: 1) aclarar qué se desea obtener del material; 2) estudiar el material; 3) establecer las clases y los indicadores de ella (crear instrucciones); y, 4) ajustar las respuestas no clasificables. Se procedió al análisis sistemático del texto, identificando y agrupando temas y contenidos, clasificando y creando categorías ${ }^{(20)}$.

Se utilizó la definición de profesionalismo de Arnold y Stern (ver anexo). El anexo lista ejemplos de actitudes y conductas relacionados con los principios el profesionalismo.

Se conformó un panel de expertos, integrado por médicos de diferentes especialidades, profesores con amplia experiencia profesional y docentes de tres departamentos académicos de la Facultad. Inicialmente, cada miembro del panel identificó competencias que hacían alusión al concepto de profesionalismo y luego se discutió en grupo las diferencias hasta alcanzar un consenso. Se procedió de igual modo con los contenidos, estrategias metodológicas y evaluación de los cursos considera- 
dos en el estudio. Es decir, se estableció la confiabilidad inter-evaluador para lograr mayor precisión en las observaciones, la replicabilidad del estudio y alcanzar consensos en los hallazgos ${ }^{(20)}$.

\section{RESULTADOS}

En la EAP de Medicina Humana, los sílabos de los cursos están formulados como competencias. Sin embargo, el uso del término no se corresponde con la definición propuesta por Barriga (21) y más bien se orientan a señalar objetivos del aprendizaje; asimismo, en contenidos clasificados como conceptual, procedimental y actitudinal se hallan inmersas las competencias; por consiguiente, fueron tratadas como tales. A continuación se presenta los 22 cursos separados en dos grupos: aquellos que se llevaban a cabo en aula y los que tenían lugar en campo clínico. En las tablas 1 y 2 , el signo (*) indica las conductas vinculadas con los principios de excelencia, humanismo y responsabilidad. El número de asteriscos cuantifica la frecuencia en que se repite las competencias asociadas a cada uno de los principios del profesionalismo en cada curso evaluado.

En general, en los once cursos dictados en aula, se observa una discordancia entre competencias y la lista de clases teóricas. La excepción son los cursos de introducción a la ética, bioética y epidemiología, en los que hay clases que hacen mención a competencias, p. ej., "derechos de los pacientes, ética de la relación médico paciente y familia, ética de las relaciones interprofesionales, denuncias éticas atendidas por el Colegio Médico del Perú, los principios éticos en la investigación epidemiológica". En ciencias de la conducta aplicada a la medicina, el contenido de tres de las cuatro unidades que comprende el curso tiene fundamentos de humanismo, así como la totalidad de sus seminarios y prácticas.

En los cursos que se desarrollan en campo clínico se observa también discordancia entre competencias y con-

Tabla 1. Principios del profesionalismo.

\begin{tabular}{cccc} 
Cursos en aula & Excelencia & Humanismo & Responsabilidad \\
Introducción a la ética & $* *$ & $* *$ & $*$ \\
Conocimiento científico e investigación & $* *$ & $*$ & - \\
Estadística y demografía en salud & $* * *$ & $*$ & - \\
Ciencias de conducta aplicadas a medicina & $* *$ & $* * *$ & - \\
Proyecto de investigación & $* *$ & $*$ & - \\
Farmacología & $* * *$ & $*$ \\
Salud comunitaria & $*$ & - & $*$ \\
Sexualidad humana & $* * *$ & $* *$ & - \\
Bioética & $*$ & $*$ & - \\
Epidemiología & $*$ & - & \\
Gerencia en salud & $* * *$ & & $*$ \\
\hline
\end{tabular}

Tabla 2. Principios del profesionalismo

\begin{tabular}{cccc} 
Cursos en campo clínico & Excelencia & Humanismo & Responsabilidad \\
Introducción a la clínica & $* *$ & $*$ & $*$ \\
Diagnóstico por imágenes & $* *$ & $* *$ & $*$ \\
Medicina interna & $* * *$ & $* * *$ & $* *$ \\
Semiología psiquiátrica y & $* * *$ & $* *$ & - \\
psiquiatría & & $* *$ & - \\
Cirugía humana & $* * *$ & $* *$ & $*$ \\
Medicina física y rehabilitación & $*$ & $* *$ & $*$ \\
Medicina legal & $* *$ & $* *$ & $*$ \\
Ginecología & $* *$ & $* * *$ & - \\
Obstetricia & $* * *$ & $* *$ & - \\
Pediatría & $* * *$ & $* * *$ & $*$ \\
Atención integral en comunidad & $* *$ & & $*$ \\
\hline
\end{tabular}

tenidos de las clases teóricas. Así, en cirugía los capítulos mencionan conductas bajo el encabezado de competencias actitudinales, p. ej., "puntual, responsable, ético, trabaja en equipo y con decisión, respetuoso, solidario, honesto, dedicado, presencia limpia y adecuada, discreción, consideración, comunicación oral y escrita"; algunas son propias del capítulo: aparato locomotor: "el alumno explica al paciente sobre su fractura, cómo se va a realizar el transporte, cómo le van a vendar o realizar la férula y la tracción, y le da valor para enfrentar su problema"; técnica quirúrgica y anestesiología: "asume la responsabilidad del cuidado apropiado y manejo humanitario de los animales de cirugía experimental"; neurocirugía: "discute los aspectos éticos ligados a la práctica médica en un servicio neuro-quirúrgico". Sin embargo, las clases teóricas no aluden a la enseñanza de tales conductas. Ginecología incluye en su primera clase: ética e historia clínica en ginecología.

Al considerar las estrategias metodológicas, los 22 cursos incluyen clases teóricas refiriéndose a ellas con diversa terminología; y prácticas, que como hemos mencionado se pueden agrupar en dos: aquellas que tienen lugar en aula y las que se dan en campo clínico. 
Adicionalmente, los sílabos incluyen otras estrategias metodológicas tales como posters; proyectos de investigación; seminarios y seminarios talleres; grupos formativos (promover el desarrollo personal, habilidades sociales y de comunicación así como las relaciones interpersonales y un mayor conocimiento de sí mismo); actividades educativas de proyección a la comunidad (charlas educativas sobre sexualidad humana, enfermedades de trasmisión sexual, sida, planificación familiar); monografías; encuestas; mesa redonda; conversatorio clínico patológico y terapéutico; foros; video-foros; discusión de casos clínicos; aula virtual; revista de revistas; búsqueda de información en Internet.

Principios del profesionalismo. El curso de Medicina interna, en las prácticas clínicas señala que: "el profesor encargado además de ejercer labor de tutoría en aspectos cognitivos y adquisición de destrezas: deberá motivar a los alumnos para desarrollar una actitud humanista y ética frente al paciente". En Seminario de medicina integrada (Aprendizaje basado en problemas y en su solución) hace alusión a excelencia y humanismo: "Tomar decisiones según la mejor evidencia revisada en aspectos de diagnóstico, tratamiento, pronóstico y manejo integral del caso clínico (competencia técnica), sin ignorar los valores éticos, creencias y patrones culturales del paciente (respeto). Finalmente se debe buscar el desarrollo de habilidades interpersonales, es decir, en trabajo de grupo, que lo ejerciten a reconocer, a discrepar y polemizar para lograr acuerdos y consensos (honor e integridad). En Prácticas de prevención de la salud aluden a responsabilidad: "Es tarea obligatoria que el estudiante eduque al paciente en algún tema específico relacionado fundamentalmente con la promoción y prevención de su salud (Salud pública)".

Cabe mencionar que medicina interna señala la importancia del modelo del rol en el aprendizaje de conductas deseables: "Inculcar al alumno a través de la enseñanza y la conducta ejemplar del docente, actitudes éticas de la relación humana con el enfermo, la mejora en la calidad, el incentivar el interés por la investigación y el trabajo en equipo".

En ginecología y obstetricia, las estrategias metodológicas mencionan aprendizaje basado en problemas, que consideran actividades auto-dirigidas y búsqueda de información (concepto de educación continua) En este proceso desarrollan habilidades de relación interpersonal: "trabajan de manera colaborativa en pequeños grupos, compartiendo en esa experiencia de aprendizaje la posibilidad de practicar y desarrollar habilidades, de observar y reflexionar sobre actitudes y valores que en el método convencional expositivo difícilmente podrían ponerse en acción” (humanismo). En ambos cursos, a diferencia del resto de cursos revisados, señalan explícitamente que: "El alumno dentro del desarrollo del curso, vivirá la experiencia que le permita practicar la conducta señalada en las competencias, lo cual facilitará la integración del aspecto afectivo con los aspectos declarativos (cognoscitivos), con la práctica del procedimiento o aspecto procedimental (habilidades y destrezas psicomotoras) y con los aspectos ético-actitudinales del alumno en la ejecución del procedimiento (aspecto valorativo)". Asimismo, se señala que el aprendizaje de la conducta profesional tiene lugar en el encuentro interpersonal con el paciente.

Con respecto a la evaluación, el plan de estudios de la EAPMH incluye evaluaciones para certificar la competencia de los futuros médicos. Todos los cursos evalúan el aprendizaje teórico a través de exámenes escritos cuyo porcentaje en la nota final va desde 30 (ciencias de la conducta aplicadas a la medicina y salud comunitaria) hasta 60 (diagnóstico por imágenes). El porcentaje restante corresponde a nota de prácticas y otras actividades complementarias. Cabe mencionar que la evaluación de práctica considera además de la práctica propiamente dicha un examen de la misma señalado explícitamente en ocho cursos.
Medicina interna tiene un formato de evaluación y algunos principios del profesionalismo estarían implícitos entre sus criterios: "conoce los derechos del paciente" (humanismo) y "conoce los aspectos médico-legales del ejercicio profesional" (responsabilidad).

Ginecología consigna una cartilla de evaluación de las experiencias de aprendizaje basado en competencias. La número 3. "Explicó el procedimiento y la razón de hacerlo"; y el número 20. "Terminado el procedimiento informó a la paciente del resultado, le agradeció la colaboración y se despidió". Ambos criterios se relacionan con respeto (humanismo). Adicionalmente, como criterio de evaluación de la práctica propiamente dicha mencionan: "1. Se presenta con respeto ante la paciente asegurando un trato cálido y solicitándole evacúe la vejiga; 9. Explica al paciente el diagnóstico, evolución y pronóstico; 10. Conoce y cumple los aspectos médico legales del ejercicio profesional". Las dos primeras se relacionan con humanismo y la última con excelencia y responsabilidad.

En Obstetricia, la cartilla de evaluación de las experiencias de aprendizaje incluye entre sus criterios: "5. Verificó que la paciente no presente ningún riesgo materno fetal; 6. Verificó la normalidad del trabajo de parto". Ambas se relacionan con disminuir el error y mejorar la seguridad del paciente (la mejora continua en la calidad de la atención) que tienen que ver con el principio de la excelencia. "7. Se le informó a la paciente en cuanto a los eventos que se iban a suceder", relacionado con respeto (humanismo). "8. Se dio soporte emocional a la paciente durante el trabajo de parto" y "9. Se le permitió la compañía del esposo durante el expulsivo". Ambos criterios implican compasión (humanismo). Los criterios de evaluación de la práctica propiamente dicha en obstetricia incluyen: "1. Se presenta con respeto ante el paciente; 9. Explica al paciente el diagnóstico, evolución y pronóstico; 10. Conoce y cumple los aspectos médico legales del ejercicio profesional". Las 
dos primeras se relacionan con respeto (humanismo) y el último con comprensión legal (excelencia) y responsabilidad.

En resumen, se evaluó la presencia del profesionalismo (excelencia, humanismo, responsabilidad y altruismo) en el plan de estudios de la EAP de Medicina Humana de la Facultad de Medicina, UNMSM. Se excluyó el internado por considerar que amerita otro estudio. Los principios del profesionalismo estuvieron presentes en 22 de los 38 cursos (del primero al sexto), formulados en las secciones de competencias, contenidos, estrategias metodológicas y evaluación de los sílabos. Las competencias aludieron principalmente a excelencia, en 22 sílabos; en humanismo disminuyeron a 19 y en responsabilidad hasta 10; y el altruismo no fue mencionado en absoluto. Se observó una discordancia entre competencias y listado de clases teóricas en la mayoría de cursos; sin embargo, las estrategias metodológicas en medicina interna mencionaban explícitamente tutoría y modelo de rol del docente para el aprendizaje de conductas positivas; asimismo, en ginecología y obstetricia, se señaló que se facilitará la práctica de las conductas mencionadas en las competencias, integrando aspectos cognitivos, habilidades y destrezas psicomotoras con las ético-actitudinales; en ciencias de la conducta aplicadas a la medicina consideraron los grupos formativos orientados a promover el auto-conocimiento y desarrollo de habilidades de relación y comunicación. Varios cursos mencionaban aprendizaje basado en problemas. El método de evaluación del aprendizaje fue el examen de conocimiento, cuyo porcentaje en la nota final varió entre 30 y $60 \%$, correspondiendo el porcentaje restante a la calificación del docente. Cabe mencionar que los cursos de medicina interna, ginecología y obstetricia proporcionaban formatos de evaluación y algunos de sus criterios evaluaban conductas profesionales deseables.

\section{DISCUSIÓN}

En nuestro estudio, los principios del profesionalismo estuvieron presentes en más de la mitad (58\%) de los sílabos, reflejando así la preocupación de la EAP de Medicina Humana por la formación en las cualidades y conductas positivas que postula la misión de la universidad: "formar profesionales del más alto nivel técnico y científico, son integrales, éticos, humanistas, críticos, respetuosos de los derechos humanos y el medio ambiente".

\section{Contexto de la educación médica y modelos de rol}

En la formación del médico, Markakis y cols. consideran que el proceso de socialización y la forma cómo los estudiantes aprenden e internalizan valores, actitudes y conductas humanas, observando y trabajando con otros miembros del equipo de salud resultan de la máxima importancia. Advierten que la falta de atención explícita a los valores del humanismo y profesionalismo crea ambientes de trabajo que son negativos para una buena atención médica. Asimismo, el rol del estudiante en la jerarquía del equipo no tiene en cuenta sus necesidades psicológicas de: 1) autonomía, tener la libertad de tomar iniciativa y sentir que su conducta es voluntaria; 2) pericia, sentirse eficaz y capaz de alcanzar el resultado esperado; 3) pertenencia, sentir respeto e interés por otros y a su vez sentirse respetado y cuidado ${ }^{(22)}$.

La importancia del equipo médico en moldear valores, actitudes y emociones en situaciones difíciles y problemáticas de la atención del paciente y la medicina hospitalaria, es enfatizada por Feudtner y cols. en su encuesta a estudiantes de medicina del 3er y 4to año de seis escuelas norteamericanas, entre 1992 y 1993. Respondieron haber hecho algo no ético (58\%); haber maltratado a un paciente (52\%); y una de ambas (80\%). La mayoría (98\%) escuchó al médico referirse al paciente con desprecio; fueron testigos de con- ducta no ética en miembros del equipo (61\%) y de estos se sintieron cómplices (54\%). Muchos se sintieron insatisfechos con su conducta y formación ética: experimentaron malestar o sentimiento de culpa por algo que hicieron (67\%); y consideraron que algunos de sus principios éticos se había perdido o erosionado (62\%). En una minoría importante la exposición a tales dilemas coincidió con deterioro de su moral. Así, en aquellos que fueron testigos de conductas no éticas fue mayor la probabilidad de que adoptaran la misma conducta por temor a una baja calificación o a una mayor dificultad en adaptarse al equipo; y, que evaluaran más negativamente su desarrollo ético. La alta prevalencia de actitudes y conductas como maltratar pacientes y considerar adecuados los comentarios despreciativos significaría que son aceptables para muchos médicos. Aún más preocupante son los estudiantes que respondieron que sus principios éticos fueron erosionados mas no estaban disgustados con su desarrollo ético, como si aceptaran que llegar a ser médico exige esa transformación del carácter. Esto sugiere que la conducta profesional del estudiante puede mermar a lo largo de sus años formativos ${ }^{(23)}$.

Existe una amplia gama de aspectos éticos a considerar en la enseñanza aprendizaje de la medicina. Así, Reiser señala que la relación que se establece entre profesores, pacientes, pares y escuela, y los valores que moldean la conducta, tienen una gran influencia en determinar la clase de médico que serán los estudiantes. La relación con el maestro en el arte de la medicina consignada en el Juramento hipocrático simboliza el compromiso del aprendiz a defender el honor de la familia médica y asegura que aprenderá y adoptará los principios éticos que gobiernan el ejercicio de la medicina. El imperativo ético es que los profesores deben tratar a los estudiantes como estos deben tratar a sus pacientes. Cita a Bosk, quien afirma: "tiende a ser tolerante y perdonar el error técnico pero intolerante y severo con el error moral". Los estudiantes 
deben aprender de sus profesores una virtud en medicina, el ser honestos, admitiendo los errores propios. En el proceso del aprendizaje los estudiantes deben sentirse en el deber de comunicar a sus instructores los límites de su conocimiento y que estos sepan lo que pueden pedirles que hagan. Así, la práctica de la sinceridad al final permite a ambas partes adherirse a la máxima hipocrática, la ética de no hacer daño.

Reiser agrega que las bases éticas de la relación estudiante paciente son: ante todo no hacer daño; información y consentimiento. En la relación del estudiante con sus pares, frente a una situación donde un compañero trasgrede normas éticas debe haber un mecanismo de apoyo porque así aprenderá a brindar ayuda al paciente tal como ellos mismos la dieron y recibieron. Finalmente, es importante una visión de la educación médica que reconozca el vínculo existente entre los múltiples aspectos de las acciones institucionales y los discursos que se sostienen en aula, lo que puede influir enormemente en el desarrollo ético de estudiantes así como del personal, cuerpo docente y la institución ${ }^{(24)}$.

En nuestro estudio las competencias aluden a conductas deseables que deben adquirir los estudiantes; sin embargo, las dificultades en su enseñanza se reflejan en discordancias entre competencias, contenidos teóricos, estrategias metodológicas y evaluación del aprendizaje. Al respecto, los hallazgos de Burack y cols. resultan ilustrativos. Los autores mencionados llevaron a cabo un estudio sobre enseñanza de la compasión y el respeto, específicamente cómo responden los docentes ante conductas problemáticas de los estudiantes. Hallaron que los primeros no responden o no hacen comentarios frente a conductas de falta de respeto, hostilidad o rudeza abierta y toma de atajo de parte de los segundos. Encontraron también suposiciones falsas que hay que cambiar y recomiendan: 1) tener en cuenta que la adultez temprana puede ser un momento de gran desarro- llo moral y cambio actitudinal; 2) que, en ausencia de un modelo de pedagogía moral efectivo, saber que tanto la teoría como la evidencia sugieren que el modelo de rol en situaciones complejas es más efectivo cuando el docente explica o narra lo que está modelando, p. ej., "Noten que siempre corro la cortina antes de examinar un paciente...", o "Lo que estoy tratando de hacer es ..." contrariamente a lo que la sabiduría convencional ha perpetuado, esto es, la creencia de que los valores son 'captados' pasivamente más que 'enseñados' activamente. Los estudiantes imitan los valores modelados por los profesores y tales atributos se enseñan mejor a través de la demostración y el ejemplo en el contexto clínico; 3) la intervención correctiva puede ser directa aunque positiva y sin juzgar. En lugar de decir "Usted faltó al respeto", podría decirse: "Usted utilizó un término que puede ser ofensivo para muchos" o "No me gusta el término usado porque ...". Para llevar a cabo las recomendaciones se requiere de cambios institucionales y quizás el tipo de modelo más importante es tratar a los estudiantes con compasión y respeto al igual que queremos que ellos traten a sus pacientes ${ }^{(5)}$.

Otro hallazgo de nuestro estudio fue la discordancia entre competencias y métodos de evaluación del curso y al respecto consideramos importante la revisión de la literatura que se presenta a continuación.

\section{Evaluación de la conducta profesional}

Tradicionalmente las escuelas de medicina vienen evaluando conocimientos y habilidades; y, a menudo, la evaluación de conductas incluyendo el profesionalismo es implícita, no sistemática y por consiguiente no adecuada; asimismo, no se da al estudiante la oportunidad de enmendarse ${ }^{(25)}$. Así, los estudiantes que presentan conductas no profesionales no pueden ser identificados en el sistema actual y serán promovidos académicamente sobre la base de un buen desempeño en exámenes solamente de conocimientos y habilidades ${ }^{(26)}$.
El estudio de Kassenbaum y Eaglen utilizó archivos del Liaison Committee on Medical Education (LCME) de escuelas que respondieron a una encuesta de acreditación entre 1993-1998. Hallaron discordancia entre los objetivos del curso y los métodos de evaluación. Solo 24,8\% de los cursos evaluaron logros no cognitivos. El aprendizaje basado en problemas para afinar la habilidad del estudiante para el razonamiento hipotético deductivo, alentar hábitos de investigación y mejorar el saber personal y las habilidades de comunicación, no fueron evaluados. En casi todas las escuelas del estudio toda la práctica clínica confió primariamente en la evaluación sumativa de habilidades clínicas llevada a cabo por docente o residente (contribuye con 50 a $70 \%$ del grado del estudiante), aunque tales calificaciones se basaban mayormente en la presentación de casos, elaboración de historias y discusión de los estudiantes ${ }^{(27)}$.

\section{Aprendizaje explícito y tácito}

Coulehan y Williams sostienen que un aspecto importante de la educación médica es la transmisión de un conjunto de creencias y valores relacionados con lo que significa ser un buen médico. Este proceso incluye componentes tanto explícitos como tácitos. La primera se refiere a las formas cómo se intenta inculcar valores expresamente y la segunda, que tiene lugar paralelamente, corresponde a lo que Hafferty denomina currículo oculto y Hundert currículo informal. Este último componente es más fuerte que el primero no solo porque es reforzado más frecuentemente sino porque se relaciona más con 'hechos' que con 'palabras'. Por ejemplo, en relación a la compasión el currículo explícito señala: empatía, habilidades de comunicación, alivio del sufrimiento, importancia de la confianza y fidelidad, foco sobre el mejor interés del paciente. El tácito, de otro lado, señala objetividad, indiferencia, cautela y desconfianza de: las emociones, del paciente, compañía de seguros, administradores, Estado. Adicionalmente, los autores señalan que el aprendizaje 
tácito favorece tres características que dificultan que un médico sea benevolente: 1) desapego, el estudiante durante su formación se torna cínico hacia valores como ternura y virtud porque aprenden que pueden sobrevivir mejor desarrollando una mentalidad de 'nosotros contra ellos'; 2) sentimiento de tener derecho a ingresos altos, prestigio y poder social; y, 3) profesionalismo no reflexivo, es decir, un sistema de creencias con el que los médicos se adhieren a los valores tradicionales aunque (relativamente) no perciben que ellos basan mucho de sus conductas en creencias contrarias a tales valores ${ }^{(28)}$.

Finalmente, el altruismo como principio del profesionalismo no se menciona en el plan de estudios de la EAP de medicina humana de la UNMSM. Al respecto consideramos relevantes los comentarios de Hafferty: "El altruismo, que se ha considerado nuclear y parte de la definición, ha desaparecido del léxico del profesionalismo. Aunque no ha sido declarado caduco, la nueva generación de estudiantes, residentes y médicos es cautelosa ifrente al mismo? "El altruismo ha llegado a ser sospechoso. Es algo que los pacientes manipuladores y los médicos mayores utilizan para explotar al estudiante cándido e impotente; lejos de fortalecerlos parece que los hace más débiles y vulnerables". $\mathrm{El}$ autor plantea la pregunta ipuede haber un real profesionalismo sin altruismo? Responde que no sabe y que el término se seguirá usando pero no en su connotación tradicional. Al mismo tiempo, debe haber formas de exponer al aprendiz en temas de deber y responsabilidad sin provocar temor de estar iniciándose en una carrera de 'víctima'. Adicionalmente, señala que la educación médica tradicional, en su mayor parte, ha relegado la enseñanza del profesionalismo al currículo no formal. Una razón de ello está en la creencia de que los médicos llegan a ser profesionales no en virtud de una instrucción determinada sino simplemente por haber completado los requisitos del proceso educativo. Respecto al modelo de rol, hasta la fecha, los atributos del médico como persona profesional han sido más un tema de fe que de evidencia. A ello se suma que el escenario de la formación médica ha sido pintado como un lugar inhóspito. Se conoce de estudios en estudiantes de medicina que evidencian un cambio del idealismo al cinismo; la existencia de una subcultura invisible y desconocida para el cuerpo docente; y, el impacto negativo en el desarrollo moral y el abuso de que son objetos a lo largo de la educación médica ${ }^{(29)}$.

Una limitación del presente estudio es que la revisión solamente de los sílabos resulta insuficiente para una visión más completa de la formación profesional en nuestra escuela. Afirmaciones tales como: "El profesor encargado deberá motivar a los alumnos para desarrollar una actitud humanista y ética frente al paciente"; o "El alumno dentro del desarrollo del curso, vivirá la experiencia que le permita practicar la conducta señalada en las competencias", no tienen en cuenta la realidad del contexto donde tiene lugar el aprendizaje y cuya complejidad ilustra la revisión de la literatura. Asimismo, cabe señalar que el tema del profesionalismo es una preocupación constante en la EAPMH y muestra de ello son las constantes revisiones curriculares. Adicionalmente, el Instituto de Ética en salud viene ocupándose de los aspectos éticos del profesionalismo a través de sus diversas publicaciones.

\section{ANEXO: PRINCIPIOS DEL PROFESIONALISMO}

\section{La excelencia}

Es el compromiso a alcanzar la excelencia a través de un esfuerzo consciente y continuo que excede las expectativas ordinarias. Comprende:

- Compromiso con la competencia en conocimiento y habilidades técnicas.

- Comprensión ética y legal.

- Habilidades de comunicación.
- Concepto de educación continua (actividades auto-dirigidas y habilidades de búsqueda de información que surgen en aquellos con una motivación sostenida de aprender y reconocer sus propias necesidades de aprendizaje).

- Mejora continua en calidad de la atención: disminuir errores, mejorar la seguridad del paciente, disminuir el derroche en los recursos de salud, generar medidas de calidad de la atención y utilizarlos en evaluaciones rutinarias de desempeño de todos los participantes (ABIM, 2002).

- Promoción del conocimiento científico y tecnológico. Defender estándares científicos, promover la investigación y producir nuevo conocimiento basados en evidencia científica y experiencia médica. Salvaguardar y mantener la integridad del conocimiento médico y tecnología y asegurar la integridad en el uso de los mismos.

\section{El humanismo}

Consiste en:

- Respeto: se refiere a interesarse por otra persona con estima, deferencia y dignidad. En medicina respeto es "compromiso a honrar elecciones y derechos personales, respecto en su atención médica" (ABIM, 1992). Incluye sensibilidad y simpatía hacia la cultura, sexo, edad y limitaciones (ACGME, 1999). Se ha dicho que es la esencia del humanismo, dado que señala el reconocimiento del valor del ser humano individual así como su sistema de creencias y valores. Respetar es ser justo con los pacientes y exige confidencialidad, privacidad y consentimiento informado.

- La empatía es una función cognitiva, habilidad para comprender el punto de vista, experiencias internas y sentimientos de otra persona sin involucrarse emocionalmente. Ser comprendido es una necesidad humana básica y medular en la relación médico paciente aunque va más allá 
e incluye la capacidad de comunicar dicha comprensión. Es diferente de la simpatía que puede interferir con la necesaria objetividad en la atención médica.

- Compasión es un sentimiento o emoción movido por el sufrimiento del otro y el deseo de aliviarlo (Diccionario Oxford, 1989). En medicina implica una apreciación que la enfermedad engendra necesidades especiales de alivio y auxilio pero sin involucrarse mucho afectivamente porque podría socavar la responsabilidad profesional hacia el paciente; asimismo, la expresión de dicha apreciación a través de una adecuada comunicación y acción.

- Honor e integridad se refiere a "ser justo y veraz, mantener la palabra, el compromiso y ser honesto". Estas cualidades se deben observar en las relaciones profesionales y se aplica a una variedad de actividades incluyendo atención del paciente, tareas académicas y en investigación. Ejemplos de conductas de estas cualidades: admitir errores, señalar errores de otros, dar crédito adecuado al trabajo de otros. Ejemplos negativos: robar, hacer trampa, adulterar datos, falsificar, suplantar.

\section{La responsabilidad}

- Central en nuestra definición de responsabilidad están las actividades de autorregulación a través de las cuales los médicos son responsables de su competencia profesional, conducta ética y legal hasta en lo económico. Establecer estándares para los miembros actuales y futuros de la profesión, comprometerse al escrutinio interno, aceptar el escrutinio externo, rehabilitación y disciplina de miembros que no alcanzan estos estándares.

- Reconocer que los conflictos de intereses son inevitables en el ejercicio de la medicina. El creciente número de acuerdos financieros con aseguradoras, farmacéuticas, esfuerzos para atender costos, afiliación de médi- cos a laboratorios farmacéuticos y la evaluación de centros médicos garantizan esta atención. Evitar tales conflictos aunque es rara vez posible, la mayoría de las organizaciones recomiendan información y monitoreo externo de tales relaciones para asegurar que la percepción de parte de pacientes y pares no sean influenciados negativamente.

- Servicio público. Implica la libre aceptación del deber de servir a pacientes y público, significa la defensa de pacientes individuales de tal manera que ellos reciban la mejor atención posible, el acceso a la salud de todos pero en especial de poblaciones tradicionalmente desatendidas. Comprende además aproximaciones sistemáticas para promover, mantener y mejorar la salud de individuos y poblaciones a través de la consejería de pacientes y sus familiares, y a través de la educación y acción pública (Responsabilidad con la comunidad).

\section{El altruismo}

Puede estar incluido en el dominio de la excelencia (demandando lo mejor para los pacientes); responsabilidad (evitando intereses personales); o humanismo (conducta desinteresada). El altruismo en medicina demanda que los mejores intereses del paciente más que los del médico guíen la conducta.

La literatura coincide en que el altruismo se expresa en situaciones específicas como aquellas conductas orientadas a beneficiar a otro aunque no está definido si tal acción debe significar para el actor algún riesgo para su bienestar, algún sacrificio o renuncia a la auto-preservación. Sin embargo, la esencia del altruismo permanece como acciones orientadas a acrecentar el bienestar de otros, particularmente de aquellos en necesidad (Piliarin y Charing, 1990; Barton, 2002). Se halla en la compasión como un sentimiento profundo de conexión con otros (McGaghie y col, 2002).

\section{REFERENCIAS BIBLIOGRÁFICAS}

1. Hensel WA, Dickey NW. Teaching professionalism: passing the torch. Acad Med. 1998;73:865-70.

2. Perales A, Mendoza A, Ortiz P. El mercado profesional como determinante de inconducta médica. An Fac med. 2000;61(3):207-18.

3. American Board of Internal Medicine. Project Professionalism; 1995. Disponible en: http://www. abim.org/pubs/profess.pdf

4. Profesionalismo médico en el Nuevo milenio: un estatuto para el ejercicio de la medicina. Rev Med Chile. 2003;131:457-60.

5. Burack JH, Irby DM, Carline JD, Root RK, Larson EB. Teaching compassion and respect. Attending physicians' responses to problematic behaviors. J Gen Intern Med. 1999;14:49-55.

6. Relman AS. Education to defend professional values in the new corporate age. Acad Med. 1998;73:1229-33.

7. Risco de Domínguez G. Educar por competencias a los profesionales de la salud para transformar la salud. Rev peru med exp salud publica. 2014;31(3):413-6.

8. Cohen JJ. Leadership for medicine's promising future. Acad Med. 1998;73:132-7.

9. The Medical School Objectives Writing Group. Learning objectives for medical student education-guidelines for medical school: report I of the Medical School Objectives Project. Acad Med. 1999;74:13-8.

10. Swing SR. The ACGME outcome project: retrospective and prospective. Med Teacher. 2007;29:648-54.

11. Baldwin DC, Daugherty SR. Using surveys to assess professionalism in individuals and institutions. En: Stern DT, Editor. Measuring medical professionalism. New York: Oxford University Press. 2006:95-116.

12. Shrank WH, Reed VA, Jernstedt C. Fostering professionalism in medical education. A call for improved assessment and meaningful incentives. J Gen Intern Med. 2004;19:887-92.

13. Lynch DC, Leach DC, Surdyk PM. Assessing professionalism for accreditation. En: Stern DT, Editor. Measuring medical professionalism. New York: Oxford University Press. 2006:265-79.

14. Mish DA. Evaluating physicians' professionalism and humanism: the case for humanism "connoisseurs". Acad Med. 2002;77:489-95.

15. Arnold L. Assessing professional behavior: yesterday, today and tomorrow. Acad Med. 2002;77:502-15.

16. Swick HM, Szenas P, Danoff D, Whitcomb ME. Teaching professionalism in undergraduate medical education JAMA. 1999;282:830-2.

17. Woolliscroft JO, Howell JD, Patel BP, Swanson DB. Resident-patient interaction: the humanistic qualities of internal medicine residents assessed by patients, attending physicians, program supervisors and nurses. Acad Med. 1994;69:216-24.

18. Hemmer PA, Hawkin AR, Jackson JL, Pangaro $\mathrm{LN}$. Assessing how well three evaluation methods detect deficiencies in medical students ' professionalism in two settings of an internal medicine clerkship. Acad Med. 2000;75:167-73.

19. Arnold L, Stern DT. What is medical professionalism? En: Stern DT, Editor. Measuring medical professionalism. New York: Oxford University Press. 2006:15-37.

20. Goode WJ, Hatt PK. Métodos de investigación social México: Ed. Trillas. Decimoquinta reimpresión 1988. 
21. Barriga C. En torno al concepto de competencia Educación. Mayo 2004;1(1):43-57.

22. Markakis KM, Beckman HB, Suchman AL, Kranke RM. The path to professionalism: cultivating humanistic values and attitudes in residency training Acad Med. 2000;75:141-50.

23. Feudtner C, Christakis DA, Christakis NA. Do clinical clerks suffer ethical erosion? Students perception of their environment and personal development. Acad Med. 1994;69:670-9.

24. Reiser SJ. The ethics of learning and teaching medicine. Acad Med. 1994;69:872-6.

25. Ginsburgh S, Regehr G, Hatala R, McNaughton N, Frohna A, Hodges B, Lingard L, Stern D. Context, conflict and resolution: a new conceptual framework for evaluating professionalism. Acad Med. 2000;75(10):S6-S11.

26. Phelan S, Obenshain S, Galey WR. Evaluation of non-cognitive professional traits of medical students. Acad Med. 1993;68:799-803.

27. Kassenbaum DG, Eaglen RH. Shortcoming in the evaluation of students' clinical skills and behaviors in medical school. Acad Med. 1999;74:841-9.

28. Coulehan J, Williams PC. Vanquishing virtue: the impact of medical education. Acad Med. 2001;76:598-605.

29. Hafferty F. Measuring professionalism: a commentary. En: Stern DTT, Edit. Measuring medical professionalism. New York: Oxford University Press. 2006:281-306.
Artículo recibido el 19 de noviembre de 2014 y aceptado para publicación el 9 de julio de 2015.

Conflicto de intereses:

Los autores declaran que no existe conflicto de intereses.

Estudio sin fuente de financiación.

Correspondencia:

Dra. Cecilia Sogi

Correo electrónico: rurisogi@yahoo.com 